\title{
Valuing diversity in value assessment: introducing the PhRMA Foundation Health Disparities Challenge Award
}

Eberechukwu Onukwugha, MS, PhD

Throughout the entire history of the field, value assessment researchers have been working to ensure that health care decision makers are equipped with the best evidence and most rigorous models available.

More recently, partially as a result of the public health and social challenges that defined 2020, more members of the value assessment community have begun to ask difficult questions about the field's current approach to issues of health equity-and whether the community has done enough to ensure all voices are appropriately represented in decision making and evidence generation. It is becoming increasingly clear that we cannot fully understand value in health care without first examining the factors that underlie and reinforce health disparities in the United States.

A successful examination of value in health care will require that the value assessment community rethink present conceptions of value and identify the gaps where the empirical research on value assessment falls short in terms of its attention to health disparities, social determinants of health, and structural inequities.

As a first step to filling these gaps, last year the Pharmaceutical Research and Manufacturers of America (PhRMA) Foundation launched a new annual award program soliciting research proposals that evaluate how the field of value assessment can better serve diverse populations and address the drivers of health disparities. Candidates were asked to submit proposals that advanced solutions to the following question: How can value assessment methods and processes better account for populations that are typically underrepresented in research and drivers of health disparities?

The following series will present the 4 winners of this innovative award mechanism. In their own way, each winning team has made a substantial contribution to what I expect will be a growing movement to make the value assessment field more inclusive and better attuned to the needs of diverse populations.

In first place, Eline M. van den Broek-Altenburg, Jamie S. Benson, and Adam J. Atherly of the Larner College of Medicine at the University of Vermont, Burlington, along with Stephane Hess of the Choice Modelling Centre \& Institute for Transport Studies at the University of Leeds in the United Kingdom conducted an analysis of COVID-19 vaccine preferences among underrepresented populations. Using latent class analysis, the team built a model identifying key factors underlying the disparities in COVID-19 vaccination and found that health care interventions intended to reduce health disparities that do not reflect the underlying values of individuals in underrepresented populations are unlikely to be successful.

In second place, Surachat Ngorsuraches of Auburn University

\author{
Author affiliation \\ Eberechukwu Onukwugha, MS, PhD, \\ Associate Professor of Pharmaceutical \\ Health Services Research, University of \\ Maryland School of Pharmacy, Baltimore \\ AUTHOR CORRESPONDENCE: \\ Eberechukwu Onukwugha, \\ eonukwug@rx.umaryland.edu \\ J Manag Care Spec Pharm. \\ 2021;27(9-a) \\ Copyright $\odot 2021$, Academy of Managed \\ Care Pharmacy. All rights reserved.
}

in Alabama describes 2 approaches to empirically address health equity in value assessment: using a discrete choice experiment to elicit preferences from individuals on preferences value attributes with a latent class model to derive the value of equity and using a flexible choice model to value health equity. Both approaches aim to capture patient preferences and ensure the systematic consideration of equity in health care decision making.

For third place, 2 teams tied for the foundation's award. Leticia R. Moczygemba, Carolyn M. Brown, and Michael Johnsrud of the Texas Center for Health Outcomes Research \& Education (TxCORE) at the University of Texas-Austin propose a 2-pronged 
strategy to increase the diversity of populations that participate in research and address drivers of health disparities to better inform value assessment. The first part of this strategy consists of a comprehensive national campaign to inform, create buy-in, and generate excitement for participation in research. Following this, the researchers propose an expediting of current methodological initiatives to require a minimum set of patient-reported social determinants of health elements to be collected and reported in research, including clinical trials and observational studies as a way to enhance the information used in value assessment frameworks.

At the University of Florida in Gainesville, Vakaramoko Diaby, Askal Ali, Aram Babcock, Joseph Fuhr, and Dejana Braithwaite examine emerging value assessment frameworks in the United States and present examples where evidence on outcomes and preferences for value do not take into consideration diverse perspectives. They then identify possible solutions to improve existing value assessment methods and use a hypothetical case study to illustrate an alternative value assessment framework to evaluate prevention choices for women at high risk of developing breast cancer.

These proposals are an important first step towards meaningfully refocusing value assessment through a health equity lens. I am confident that, in future years, the body of research exploring the intersection of value assessment and health equity will only continue to grow. If the following proposals are any indication, this growth will be inclusive, representative, and transformative. Please take some time to review the following papers, each of which considers health equity and value assessment in a different, though no less important, context.

I hope you are as excited as I was to learn about these proposals.

\section{DISCLOSURES}

No funding supported the writing of this article. The author has received grants from BeiGene, Ltd., and Pfizer, Inc. and advisory board fees from PhRMA Foundation. 